\title{
Surgery for the rheumatoid wrist and hand
}

\author{
S H Norris
}

Surgery for rheumatoid arthritis of the hand and wrist has improved the lot of many with this condition over the past several years, and there is no doubt that surgery can make an enormous difference to the course of the disease. Pain, deformity, poor function, and cosmesis are all indications for surgery. There are, however, many patients who are denied access to surgery who would benefit from it.

\section{PATTERNS OF DISEASE}

Progression of the rheumatoid process at the wrist produces a number of recognisable deformities, which occur in a more or less constant fashion.

The disease at the wrist may affect the radiocarpal, mid-carpal, and radioulnar joints (fig 1). At the radioulnar joint the tendon of extensor carpi ulnaris has a significant role. ${ }^{1}$ In the normal wrist the tendon crosses the extensor surface of the distal ulna, but in the rheumatoid wrist it comes to lie on the volar surface of the ulna as the distal ulna begins to subluxate on the radius. Thus the tendon behaves as a flexor of the wrist rather than as an extensor. This has the effect of causing an imbalance between the radial and ulnar extensors, and the radiocarpal joint becomes pulled into radial deviation.

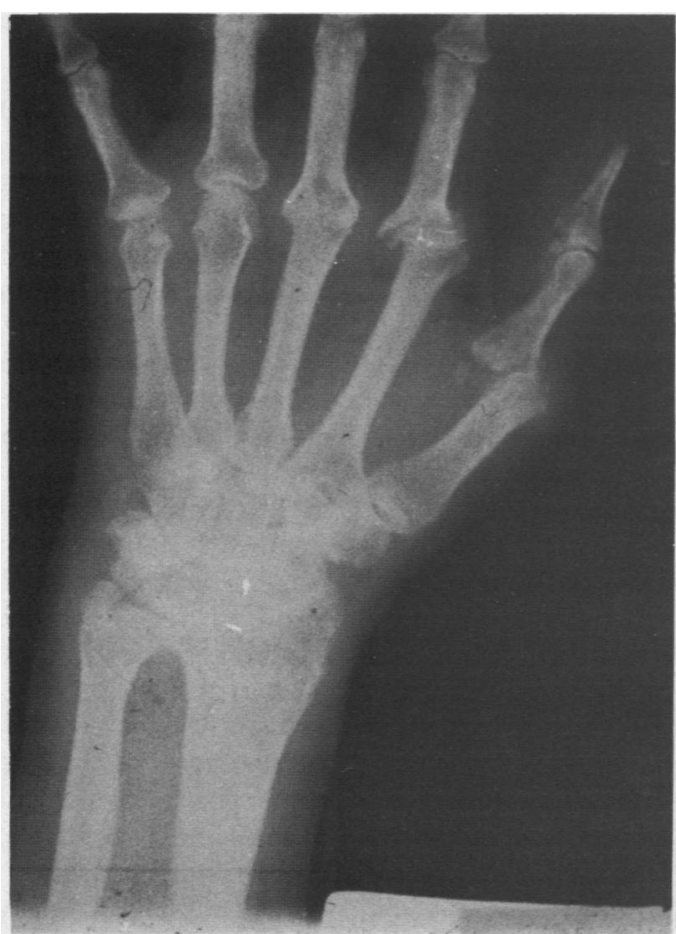

Hospital, Shefield

Figure 1 Radiograph of the wrist showing severe erosive changes and damage to the radiocarpal, mid-carpal, and carpometacarpal joints.
This deformity has grave implications for the metacarpophalangeal (MCP) joints. As the wrist becomes pulled into radial deviation so the fingers start to become pulled into ulnar deviation. This is only one of the causes of ulnar drift of the MCP joints, but a very important one, which may easily be attended to surgically. ${ }^{2}$ Furthermore, as the tendon slips from its position on the back of the ulna so it loses its role as a stabiliser of the distal ulna, permitting more dislocation. The dislocation of the ulna, together with the severe erosions on the lower end of this bone, puts the extensor tendons of the fingers at considerable risk of rupture.

This clinical picture-(a) subluxation of the distal ulna; $(b)$ radial deformity of the wrist; (c) dropped fingers from extensor tendon rupture (fig 2)-is known as the caput ulnae syndrome.

At the radiocarpal joint progressive stretching of the joint capsule together with joint erosion results in ulnar displacement of the proximal carpal bones with secondary radial deviation of the hand. At the same time the scaphoid and the lunate slip into a palmar position on the radius. In some cases the radius and the lunate become spontaneously fused. This produces a degree of pain relief, but restricts movement.

\section{CLINICAL FEATURES}

Patients with wrist disease present in a variety of ways. The most common is significant dorsal swelling resulting from extensor tendon synovitis. In most cases this will produce pain on movement of the digits. There will often be associated subluxation of the distal ulna. Wrist motion may be preserved, depending upon the degree of articular cartilage damage. Progression of the disease will result in the deformities

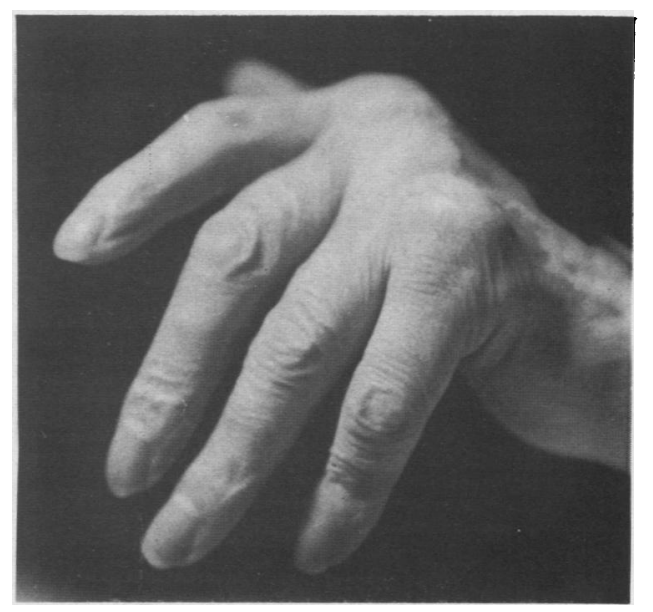

Figure 2 The extensor tendons to the middle, ring, and little fingers have ruptured. 
described above. Rupture of the extensor tendons is a grave complication.

\section{SURGICAL TREATMENT}

Both soft tissue and bony procedures are of benefit for the rheumatoid wrist

Synovectomy not only relieves pain and swelling but can also damp down the arthritis. It is indicated in early disease when conservative measures have failed. The procedure is performed through a straight dorsal incision to prevent necrosis of the skin flaps. The skin of patients with rheumatoid arthritis can be very thin, especially if the patient is being treated with steroids, and a curved incision is very susceptible to flap necrosis even if the flaps do not seem to have been undermined to any significant extent. The dorsal retinaculum is divided and reflected in both directions, rather like the pages of a book, to expose the dorsal tendons beneath.

Inflamed synovium is stripped from the tendons and often it can be seen that the synovium has eroded into the structure of the tendon, which is in imminent danger of rupture. Such areas are weaknesses in the tendon and as much synovium as possible should be removed.

At the same time as synovectomy of the extensor tendons the wrist joint is opened and synovectomy performed. A complete clearance of the wrist is impossible but, nevertheless, a proportion of the synovium can be cleared away and this will contribute to pain relief.

Reflection of the dorsal retinaculum towards the ulnar side of the wrist allows the distal radioulnar joint to be opened. The ulna is often subluxated and the end of the bone is commonly sharp, pitted, and eroded. It is easy to see how an extensor tendon already weakened by infiltrating synovium will rupture by passing over the sharp distal ulna.

\section{DARRACH'S PROCEDURE}

The patient with a dislocated ulna with pain on rotation of the wrist may benefit from an excision of the distal $1 \mathrm{~cm}$ of the ulna, known as Darrach's procedure. Although this operation has been shown by several authors to provide good relief of pain, ${ }^{3-5}$ the expected result is not always forthcoming. Newman found that in $60 \%$ of patients there was no change in wrist function and in $12 \%$ there was actually a deterioration in function, though there was relief of rest pain in $77 \% .^{6}$ Pain on rotation of the forearm was improved in $86 \%$ and the range of rotation increased in $90 \%$. Eighty eight per cent graded their result as excellent or fair.

An alternative to the Darrach procedure has recently been devised by Bowers. ${ }^{7}$ The radial aspect of the distal ulna is resected in such a way that impingement between the two bones is prevented throughout the range of rotation of the forearm. The gap left by the resected ulna may be filled either by repair of the dorsal capsule or by tendon interposition. The advantage of this procedure is that the ulnar ligament complex is not disturbed and as the distal ulna has not been totally resected the wrist does not lose its ulnar support. One of the problems with the Darrach procedure is that the carpus can slide further in an ulnar direction if the ulnar ligament constraints are removed. Bowers considered that the procedure was of value in $85 \%$ of patients operated upon.

A similar procedure described by Watson et $a l^{8}$ also entails hemiresection of the ulna, and the distal ulna is shaped to conform to the shape of the distal radius. No soft tissue interposition is used in this procedure.

Associated radial deviation of the carpus with ulnar translocation should be treated by strengthening ulnar extension of the wrist. This can be accomplished by transferring extensor carpi radialis brevis to the ulnar side of the wrist and using this tendon to stabilise extensor carpi ulnaris in a dorsal rather than a volar position.

\section{ARTHROPLASTY AND ARTHRODESIS}

In more severe disease of the wrist the surgical options are either arthrodesis or arthroplasty. The choice of surgery should not be based upon radiographic signs only as it is surprising how much radiologically apparent damage some patients can tolerate before a request is made for surgical relief.

Although several types of wrist implant have been devised, the two most commonly used are Swanson's flexible silastic implant ${ }^{9}$ and Meuli's three component metal-polythene-metal prosthesis. ${ }^{10}$ Summers and Hubbard have reviewed their experience of these two devices and concluded that both afford a pain free joint which is stable and provides a solid platform for the hand with a reasonable range of movement. ${ }^{11}$ The Meuli prosthesis perhaps provides a greater range of movement than the Swanson implant, but the technique of insertion is more critical. Both prostheses have their inherent dangers of dislocation and loosening and, in addition, there is the risk of cement failure with the Meuli device.

The results of wrist arthroplasty must be compared with the results of arthrodesis. Arthrodesis of the wrist in rheumatoid arthritis is an excellent operation. Problems of union are seldom encountered, the operation is relatively quick and safe to perform, and the results are predictable.

Many different types of wrist fusion have been described. Millender and Nalebuff's technique is perhaps the most popular. ${ }^{12}$ Stability is achieved by passing a Steinmann pin through the second or third metacarpal joint into the distal end of the radius. In many cases this is all that is required, especially if the wrist is severely eroded. Autologous bone grafting is seldom necessary and by destroying the adjacent sides of the wrist most joints can be persuaded to fuse. Arthrodesis is from the radius down to the capitate. Immobilisation of the joint in plaster for six weeks will ensure that union takes place in most patients.

Other techniques of arthrodesis include the use of staples, Kirschner wires, and plates to achieve the same results. It must be remembered that fusing the wrist of a patient with rheumatoid arthritis is not the same as performing this 
operation on a young man with post-traumatic osteoarthritis. Heavy plates are not necessary and merely make the skin incision difficult to close, increasing the risk of skin breakdown. The wrist is normally arthrodesed in a neutral position, though if both wrists are being treated it may be preferable to stiffen one joint in a few degrees of flexion to enable the patient to attend to personal hygiene.

An alternative to formal wrist arthrodesis is fusion of the radiolunate joint. This procedure is indicated for treatment of pain emanating from the radiocarpal joint if the mid-carpal joint is preserved. This joint sometimes fuses spontaneously with resolution of pain. Nalebuff and Garrod suggest that perhaps one third of patients being considered for wrist fusion might be suitable for this operation. ${ }^{13}$ Linscheid and Dobyns reported good results for 13 of 16 patients undergoing localised wrist fusion. ${ }^{14}$

\section{EXTENSOR TENDON RUPTURE}

Extensor tendon rupture is one of the major complications that can befall the rheumatoid patient and it can be both predicted and prevented.

Rupture has to be distinguished from two other conditions. The first is a failure of digit extension owing to chronic dislocation of the MCP joints and the second is the comparatively rare condition of posterior interosseous nerve entrapment.

The most commonly injured tendons are extensor pollicis longus, where it passes over Lister's tubercle, and the tendons to the little and ring fingers. The tendons to the middle and index fingers are less often ruptured.

Dorsal subluxation of the ulna associated with tenderness on resisted extension of the thumb and fingers must alert the doctor to the possibility of tendon rupture. Thickening of the dorsal synovium is present and dorsal displacement of the distal ulna is nearly always an accompanying feature.

Prevention of rupture is by dorsal tenosynovectomy (see above). Treatment of established rupture is by synovectomy and tendon transfer.

The preferred transfers for a single tendon rupture are either end-to-side repair using the next-door extensor tendon or transfer of extensor indicis proprius. End-to-side repair will provide good results but without such good individual function. ${ }^{15}$ Multiple ruptures pose a severe problem. Extensor indicis proprius is not strong enough to extend more than a single digit so an alternative must be found. Flexor sublimis from the ring finger can be rerouted to the dorsum of the hand and will provide satisfactory extension to more than one digit if so desired. Occasionally, in cases of quadruple tendon rupture, it may be necessary to use two flexor tendons. ${ }^{16}$

\section{Metacarpophalangeal joint surgery}

The MCP joints are the most common site of rheumatoid arthritis affecting the hand. Swelling of the MCP joints, ulnar drift with progressive volar subluxation of the base of the proximal phalanx, and, eventually, dislocation with an inability actively to extend the joints are the hallmarks of the condition. Apart from progressive deformity pain is a common feature. The early radiological appearances are of soft tissue swelling and erosions, progressing in late cases to dislocation and destruction of the joints.

\section{SYNOVECTOMY}

The role of synovectomy has long been a contentious issue. There is no doubt that in some patients the course of the disease can be slowed down by performing synovectomy at an early stage. It must be remembered, however, that patients not infrequently show spontaneous remission, and this affects the assessment of results.

Synovectomy is indicated for patients who present with pain and persistent swelling of the MCP joints in which there are minimal radiographic changes and little deformity. Soft tissue reconstruction with synovectomy is performed for patients who have ulnar drift with dislocation of the extensor tendons but do not have radiological erosions. Before a synovectomy is performed, however, patients should be given a trial of medical treatment for not less than three to six months. If the MCP joint synovitis settles down with this treatment regimen then surgery is no longer necessary.

Ellison, Kelly, and Flatt reviewed the results of surgical synovectomy of the digital joints in rheumatoid arthritis. ${ }^{17}$ Surgical synovectomy was performed on 390 joints in 67 patients. The mean follow up was for 4.5 years. There was a significant improvement in pain relief, with only nine patients having recurrence of pain postoperatively. Twenty nine of the 67 patients showed no progression of deformity, whereas some progression of deformity was seen in the remaining 38. Radiographic progression of the disease was seen to a moderate degree in 37 patients, of whom nine could be described as having an early synovectomy, three synovectomy at an intermediate stage of the disease, and 25 a late synovectomy. Ten patients showed marked deterioration of the radiographic appearance; five of these had undergone synovectomy at an intermediate stage and four for late disease. Of the 20 patients who showed no radiographic progression, 14 had had operations for early synovitis. The radiographic progress is therefore clearly related to the stage of the disease at which the synovectomy is performed. Recurrent synovial swelling was seen in one or more joints in 20 patients. Two thirds of the patients who showed recurrent synovial swelling were operated on at an intermediate or late stage of the disease.

The MCP joints can be approached either through a transverse incision across their dorsum joints or, alternatively, individual longitudinal incisions some 3 to $4 \mathrm{~cm}$ in length can be used. Each joint is opened on the radial side by careful division of the sagittal band, which must be carefully delineated from the underlying capsule and the band must be carefully preserved for subsequent suture.

Some $60 \%$ of the synovial membrane can be 
removed surgically. If the joint is flexed and traction applied the synovium lying in the volar recesses of the joint can be reached. It is important to curette beneath the collateral ligaments and to remove the tongue of synovium which extends proximally along the dorsum of the joint between the distal end of the metacarpal and the extensor tendon.

\section{Ulnar drift}

Harrison stressed the importance of the alignment of the middle finger in identifying ulnar drift. ${ }^{18}$ If the index and ring fingers are held in full extension any ulnar deviation is seen as the middle finger flexes and extends. After synovectomy the extensor tendons are relocated by reefing of the sagittal bands. Ulnar drift of the metacarpals without significant joint erosion is eminently treatable surgically. Relocation of the extensor tendons provides some of the necessary correction of this deformity, which can also be corrected by dividing the intrinsic tendons on the ulnar sides of the index, middle, and ring fingers from their attachment to the extensor mechanism, rerouting them across the web spaces, and reattaching them to the extensor expansion on the radial side of the middle, ring, and little fingers respectively. This is known as crossed intrinsic transfer.

The concept of crossed intrinsic transfer was introduced by Straub, ${ }^{19}{ }^{20}$ who described transfer of the tendon into the lateral band of the adjacent finger. Ellison, Flatt, and Kelly sutured the intrinsic tendon to the radial collateral ligament of the adjacent finger for fear of causing a swan-neck deformity. ${ }^{21}$ The index finger is held in a corrected position by proximal reattachment of the radial collateral ligament.

Persistent volar subluxation of the proximal phalanx may be treated by the Harrison sling technique. ${ }^{18}$ A $4 \mathrm{~cm}$ slip of extensor tendon 5 $\mathrm{mm}$ wide is detached proximally and freed distally to the distal portion of the MCP joint. This is passed through a drill hole made in the dorsal cortex of the proximal phalanx. The tendon is passed through the drill hole and sutured back on itself. This provides a strong

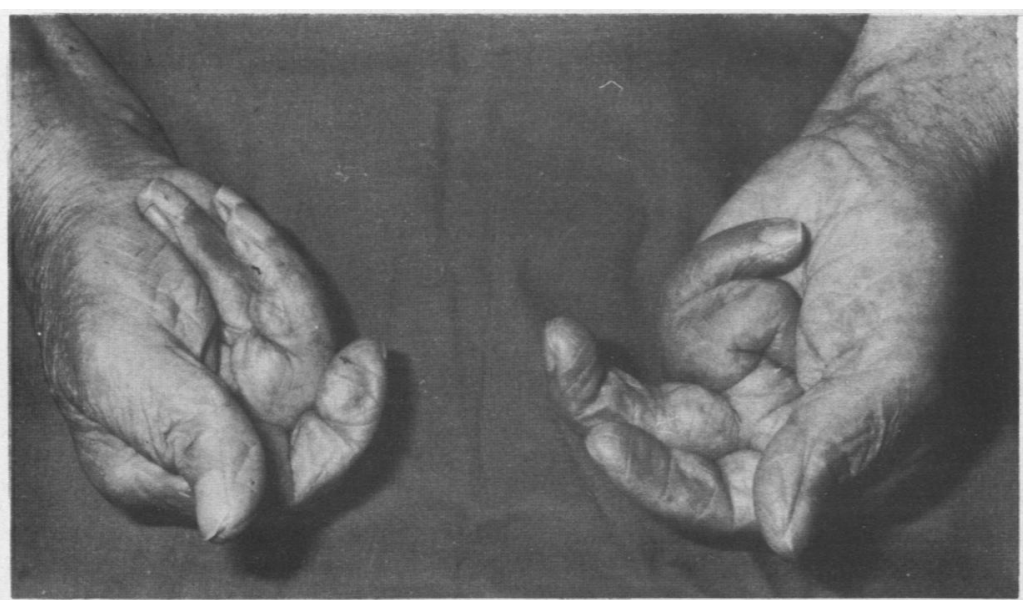

Figure 3 Severely deformed hands, which are free from pain and not suitable for reconstruction. extensor force holding the base of the proximal phalanx in its relocated position.

\section{INTERPOSITION ARTHROPLASTY}

In late cases with joint destruction and dislocation reconstruction of the MCP joints by means of silastic arthroplasty is necessary. Pain accompanied by irretrievable articular cartilage damage, volar subluxation, or dislocation is the indication for surgery. There are many patients, however, who cope very well with severely deformed hands, who are often free from pain, and for whom sophisticated surgery is irrelevant (fig 3).

The most widely used implant is the Swanson designed silicone-rubber spacer. Its function depends upon a supporting fibrous capsule developing after joint resection. Vahvanen and Viljakka performed a retrospective analysis in 32 rheumatoid patients who had undergone 107 Swanson MCP joint implants and reported an improvement in hand function in $27(84 \%){ }^{22} \mathrm{~A}$ prospective study by Bieber et al with 210 joints inserted in 55 rheumatoid hands showed initially good correction of deformity with improvement in the range of joint movement. ${ }^{23}$ When these patients were assessed in the long term at an average of 5.25 years there was some deterioration in function, though most remained better than before the operation. In the early postoperative evaluation ulnar drift improved from an average of 25 degrees to less than 5 degrees and the preoperative extension deficit improved from 56 to 10 degrees. In the long term the amount of ulnar drift had increased to 12 degrees and the extension deficit to 22 degrees. Grip strength did not seem to increase.

Silastic interposition arthroplasty is performed through similar incisions as described for synovectomy. Once the joint has been opened and the synovium excised the metacarpal head is excised through the neck. The intramedullary cavity of the proximal phalanx is breached with a sharp awl and then reamed using small hand-held reamers to clear a cavity large enough for the stem of the prosthesis. The canal of the metacarpal is reamed in a similar fashion. The correct size of implant is selected by using trial prostheses. The selected implant should be large enough to allow easy insertion and it should allow a reasonable range of flexion up to 70 degrees without dislocation of the stem.

Once all four implants have been inserted ulnar drift is corrected by crossed intrinsic transfers and tightening of the radial collateral ligament of the index finger. The Harrison loop reconstruction of the extensor tendon may alsc be performed. An abduction deformity of the little finger can be corrected by resecting a length of the tendon of abductor digiti minimi.

Swanson published his results of flexible implant arthroplasty in 3915 implants, of which 3867 were for the MCP joints and 148 for the proximal interphalangeal (PIP) joints. ${ }^{24}$ Thirty two of the joints dislocated after the operation and there was an infection in 27 , requiring removal in five. The range of movement at the MCP joints averaged 58 degrees. Most patients 
had a range of movement of between $\mathbf{4 0}$ and 70 degrees. Follow up ranged from six months to five years.

\section{Postoperative care}

The postoperative care of a hand which has undergone reconstructive surgery to the MCP joints is most important and a critical part of the procedure. For the first two weeks postoperatively the hand is supported in a hand dressing with the MCP joints in about 30 degrees of flexion, with 30 degrees of flexion at the interphalangeal joints.

After two weeks extension of the MCP joints can be maintained with a dynamic extension splint for daytime use and treatment, and the fingers should be rested at night on a simple paddle splint. After a further six weeks it is recommended that a metacarpal radial abduction splint should be used, which allows normal usage of the MCP joints but at the same time ulnar deviation of the fingers is resisted (figs 4A and $B$ ).

\section{Proximal interphalangeal joints}

Several patterns of rheumatoid disease of the PIP joints may be seen. Chronic thickening and inflammation of the joint is due to synovial proliferation, which causes gradual stretching and bulging of the capsule (fig 5). Boutonnière and swan-neck deformities occur in a proportion of patients. The radiological changes are similar to those seen in the MCP joints.

\section{SYNOVECTOMY}

Synovectomy of the PIP joints is a useful operation for proliferative synovitis that has failed to respond to medical treatment once again. It is important to give medical treatment a trial before embarking on synovectomy as often the synovial proliferation can be aborted

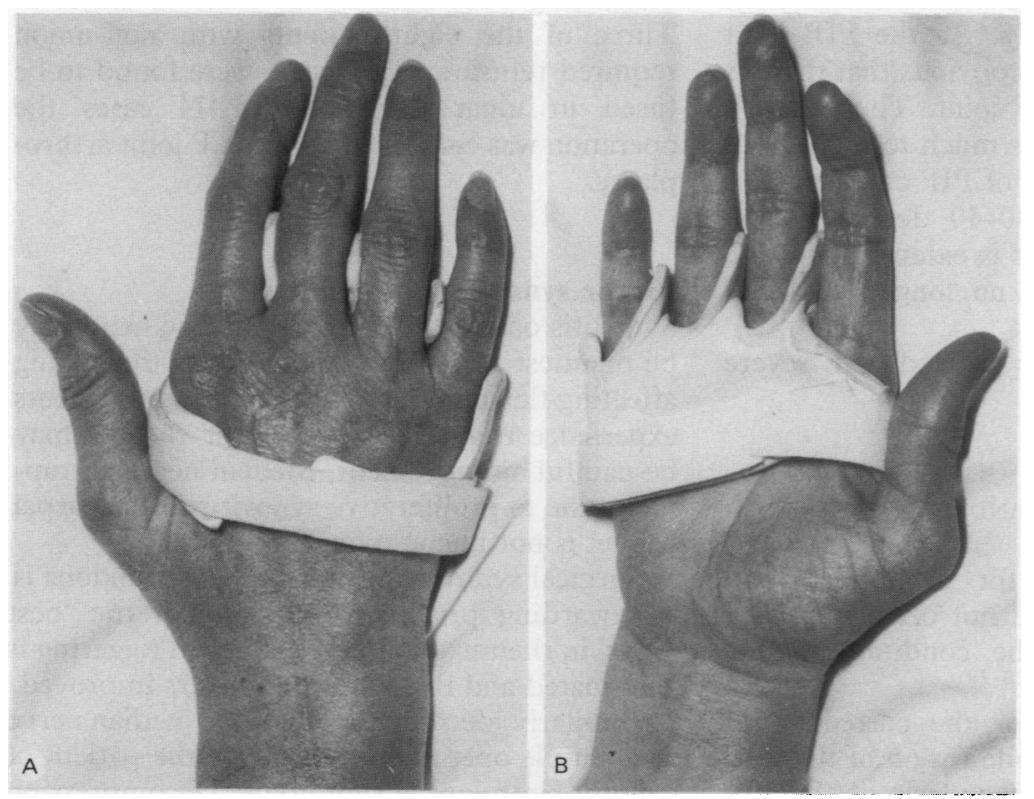

Figure 4 (A) Metacarpal radial abduction splint from the dorsum; (B) the same splint viewed from the fromt.

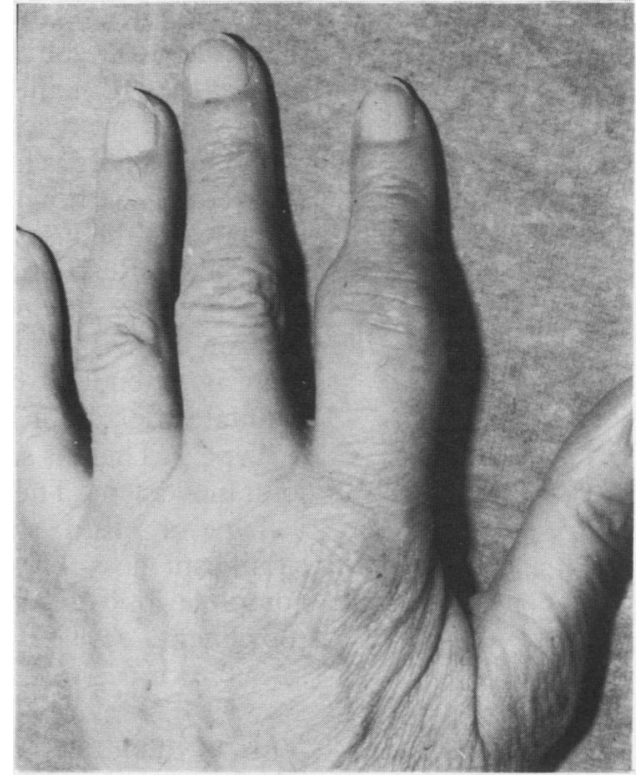

Figure 5 Chronic thickening of the proximal interphalangeal joint of the index finger.

by a good medical regimen. Injections of local anaesthetic and steroid can also be useful to control synovitis.

Synovectomy gives good relief of pain and there is usually only a slight loss of PIP joint movement. Its major disadvantage is recurrence of the synovitis. Wilde suggested that as many as $30 \%$ of patients undergoing PIP joint synovectomy have some degree of recurrence of synovial swelling. ${ }^{25}$ Ellison et al had a recurrence rate of $24 \% .{ }^{17}$ Ansell et al reviewed 115 synovectomies in 56 patients. ${ }^{26}$ In 83 fingers there was no change in the range of movement and in 17 the range improved. Pain relief made the procedure worthwhile in all the cases. A review of these cases at five years showed that there had been no radiological change in 37 joints, mild improvement in nine, and slight deterioration in $66 .{ }^{27}$ In $60 \%$ there was good relief of pain and some improvement in grip.

It is very difficult to achieve complete removal of the synovium by surgical means. The operation is performed through a posterolateral approach, and one of the collateral ligaments should be divided to remove as much of the synovium as possible.

\section{SWAN-NECK DEFORMITY}

Swan-neck deformity is characterised by hyperextension of the PIP joint with flexion of the distal interphalangeal(DIP) joint. The deformity may start at either the PIP or DIP joints.

At the DIP joint there is gradual stretching or a frank rupture of the attachment of the extensor tendon to the base of the distal phalanx. This results in a mallet deformity of the distal joint and causes an extensor tendon imbalance, which expresses itself at the PIP joint with a hyperextension deformity.

If the deformity originates at the PIP joint there is a gradual stretching of the volar plate. As the hyperextension deformity increases there is not infrequently rupture of the superficial 
flexor tendon, and this allows the hyperextension deformity to become worse. The flexion deformity at the DIP joint in these cases is secondary. Four stages are recognised:

Type 1. There is full passive movement of the PIP joint. The functional loss in these patients is related to the loss of the DIP joint extension.

Type 2. Restriction of PIP joint flexion is influenced by the position of the MCP joint. Flexion of the PIP joint is restricted if the MCP joint is extended. Flexion of the MCP joint facilitates flexion of the PIP joint.

Type 3. There is significant restriction of PIP joint movement. The joint spaces are preserved.

Type 4. End stage deformity. There is significant loss of articular cartilage with a complete loss of active and passive PIP joint movement.

\section{BOUTONNIËRE DEFORMITY}

The boutonnière deformity is characterised by a flexion deformity of the PIP joint with an extension deformity at the DIP joint. There is often an associated hyperextension deformity of the MCP joint.

Synovial proliferation within the PIP joint results in stretching of the joint capsule. The central slip of the extensor tendon stretches and the PIP joint assumes a flexed position as it is no longer supported on its dorsal aspect. The lateral bands adopt a more and more volar position and this accentuates the flexion deformity of the PIP joint. In addition, as the lateral bands become tense they cause an extension deformity of the DIP joint. As the flexion deformity of the PIP joint increases the patient compensates by hyperextending the fingers at the MCP joint in order to maintain as much independence of finger movement as possible.

In the early stages both the swan-neck and boutonnière deformities are passively correctable. As the condition deteriorates so the deformities become fixed.

Stage 1. There is a $10-15$ degree flexion deformity and the extensor lag of the PIP joint can be corrected passively. As the PIP joint deformity is corrected it is obvious that there is limited flexion of the DIP joint. The patient's functional loss is related as much to the lack of DIP flexion as to the lack of PIP extension.

Stage 2. There is a $30-40$ degree flexion deformity. The MCP joint is extended and the boutonnière deformity is no longer passively correctable.

Stage 3. The deformity is fixed with severe loss of articular cartilage.

\section{SURGERY FOR SWAN-NECK AND BOUTONNIÈRE} DEFORMITIES

Soft tissue procedures for swan-neck and boutonnière deformity are not often indicated but may be useful if the condition of the articular surfaces is good. ${ }^{28} 29$

Available procedures for the correction of swan-neck deformity include fusion of the DIP joint, dermodesis, flexor tenodesis of the PIP joint, and reconstruction of the retinacular ligaments. Distal interphalangeal joint fusion is indicated only if the swan-neck deformity originates at that joint. The joint should be fused in full extension.

Both dermodesis and the flexor tendon procedures have been popularised by Nalebuff. Dermodesis entails the excision of an ellipse of loose skin over the flexor aspect of the PIP joint and closure of the defect. Its long term results are poor, but it may be usefully combined with flexor tenodesis in which one slip of the sublimis tendon is used as a passive check-rein against full extension of the PIP joint, by being passed around a pulley in the fibrous flexor sheath and sutured back to itself.

Reconstruction of the retinacular ligament was devised by Littler. ${ }^{30}$ The ulnar lateral band is freed from the extensor tendon proximally but is left attached distally. The tendon is passed volar to Cleland's ligament to bring it to the volar side of the axis of PIP joint movement. The band is sutured to the fibrous flexor tendon sheath with enough tension to prevent extension of DIP and PIP joints. This procedure should correct both the DIP and PIP joint deformities.

Soft tissue procedures for correction of the boutonnière deformity include extensor tenotomy and reconstruction of the extensor mechanism. Extensor tenotomy is a fairly simple procedure to perform and is carried out to allow flexion of the DIP joint. This takes the tension off the proximal joint to some extent and can be effective in correcting the deformity of the PIP joint as.well as the deformity at the DIP joint.

There are many operations described to reconstruct the extensor tendon mechanism in boutonnière deformity, though the results are not predictable. Most patients respond best to either PIP joint fusion or PIP joint arthroplasty. If arthroplasty is selected it is important to reconstruct the extensor tendons as well as to replace the joints.

Granowitz and Vainio reviewed the results of PIP joint arthrodesis. ${ }^{31}$ They studied 122 operations in 65 patients. There were eight nonunions, five due to poor placement of the Kirschner wires and two in cases of amyloid. Three of the eight patients with non-union required refusion. Six fingers were found to be fused in ulnar deviation. In 14 cases the operation was combined with MCP joint arthroplasty.

\section{Flexor synovitis}

Synovitis on the flexor surface of the hand may be manifest in the flexor sheaths as thickening affecting flexion of the digits, and some patients experience triggering. The flexor sheaths may be painful on movement. Median nerve entrapment due to proliferative synovitis in the carpal tunnel is not uncommon (fig 6).

Surgical synovectomy of the flexor tendons is a rewarding procedure and one of the 'best buys' in rheumatoid hand surgery. Triggering is eliminated and flexion of the fingers improved.

Similarly, decompression of the median nerve is a simple operation, which rids the patient of painful neurological symptoms. Decompression of the nerve, however, must be accompanied by synovectomy of the flexor tendons. 


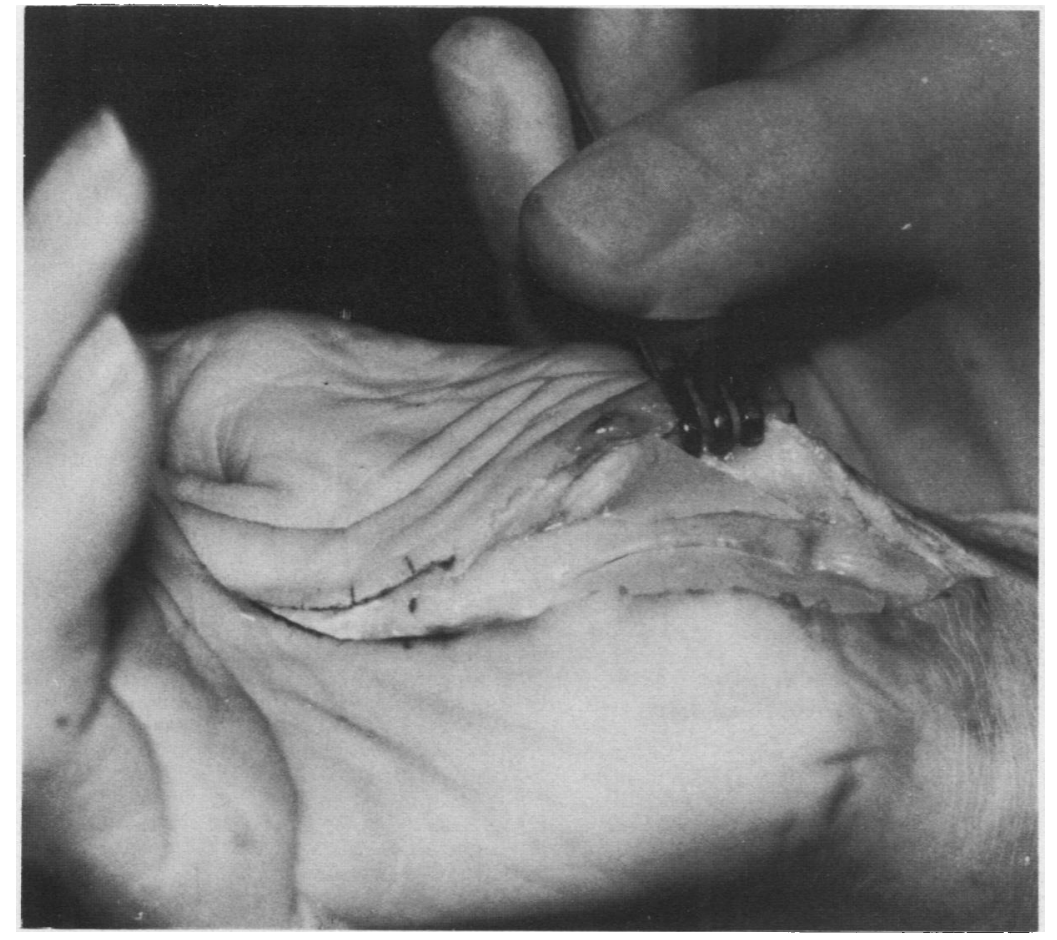

Figure 6 Severe wasting of the median nerve owing to longstanding flexior synovitis at the wrist.
SURGICAL TREATMENT OF THUMB DEFORMITY Early type 1 deformity consists of a mild degree of MCP swelling and subluxation and this is not accompanied by deformity at either the proximal or the distal joints. Synovectomy and reconstruction of the extensor tendons is required. Nalebuff has described a rerouting procedure for the extrinsic extensors which will prevent the joint from resubluxating. ${ }^{32}$ Harrison has also described a similar procedure in which extensor pollicis longus is tenodesed to the base of the proximal phalanx. ${ }^{33}$

Late subluxation of the MCP joint with cartilage erosion but without significant associated joint disease is best managed by MCP joint arthrodesis. If the CMC joint is diseased and stiff, however, replacement of the MCP joint is a better alternative as this will preserve mobility of the thumb.

Severe deformity of both the MCP and interphalangeal joints of the thumb with an intact CMC joint is an indication for arthrodesis of both the MCP and interphalangeal joints.

Whereas the boutonnière deformity is mainly at the MCP joint, swan-neck deformity is primarily due the gradual dorsal and radial subluxation of the CMC joint. As the joint erodes and subluxates the metacarpal becomes adducted. Progressive adduction of the metacarpal results in hyperextension of the MCP joint in an effort to maintain extension and abduction of the thumb.

Hemiarthroplasty of the CMC joint is the preferred operation for early deformity, though surgery is indicated only after a trial of conservative treatment including adequate splintage. Complete replacement of the trapezium in the rheumatoid patient is associated with a high incidence of dislocation.

Hyperextension of the MCP joint may be treated by MCP joint replacement. Severe adduction contracture of the CMC joint with painful fixed extension of the MCP joint is best treated by means of $\mathrm{CMC}$ joint replacement combined with fusion of the MCP joint.

Type IV deformity with weakness of the ulnar collateral ligament in early disease without significant joint destruction of the articular cartilage is treated by means of ligament reconstruction in the same way as chronic rupture is treated in non-rheumatoid patients. In later cases either arthrodesis or joint replacement is necessary.

Type $\mathrm{V}$ deformity is managed either by arthrodesis of the MCP joint or by volar capsulodesis in a position of flexion.

1 Swanson A B. The ulnar head syndrome and its treatment by implant resection arthroplasty. F Bone foint Surg [Am] 1972; 54: 906.

2 Boyce T, Youm Y, Sprague B L, Flatt A E. Clinical and experimental studies on the effect of ECRL transfer in the rheumatoid hand. Fournal of Hand Surgery 1978; 3: 390-4.

3 Rana N A, Taylor A R. Excision of the distal ulnar in heumatoid arthritis. $\mathcal{f}$ Bone foint Surg [Br] 1973; 55: 96-105.

4 Jackson I T, Milward T M, Lee P, Webb J. Ulnar head resection in rheumatoid arthritis. Hand $1974 ; 6: 172-80$.

Rasker J J, Veldhuis E F, Huffstadt A J, Nienhuis R I Excision of the ulnar head in patients with rheumatoid arthritis. Ann Rheum Dis 1980; 39: 270-4.

6 Newman $R$ J. Excision of the distal ulna in patients with theumatoid arthritis. F Bone foint Surg [Br] 1987; 69: 203-6.

7 Bowers W H. Distal radioulnar joint arthroplasty: the perextends and as it does so there is seconda flexion of the interphalangeal joint as the tension on the flexor tendon increases. 
hemiresection-interposition technique, $\mathcal{I}$ Hand Surg [Am] 1985; 10: 169-78.

8 Watson H K, Ryu J Y, Burgess R C. Matched distal ulnar resection. $\mathcal{F}$ Hand Surg [Am] 1986; 11: 812-7.

9 Swanson A B. Flexible implant arthroplasty for arthritic disabilities of the radio-carpal joint. A silicone rubber intramedullary stemmed flexible hinge implant for the wrist joint. Orthop Clin North Am 1973; 4: 383-94.

10 Meuli H Ch. Meuli total wrist arthroplasty. Clin Orthop 1984; 187: $107-11$

11 Summers B, Hubbard M J. Wrist joint arthroplasty in rheumatoid arthritis: a comparison between the Meuli and Swanson prostheses. F Hand Surg [Br] 1984; 9: 171-6.

12 Millender L H, Nalebuff E A. Arthrodesis of the rheumatoid wrist. An evaluation of 60 patients and a description of a different surgical technique. F Bone foint Surg [Am] 1973; 55: 1026-34.

13 Nalebuff E A, Garrod K J. Present approach to the severely involved rheumatoid wrist. Orthop Clin North Am 1984; 15: involved $369-80$.

14 Linscheid R L, Dobyns J H. Radio-lunate arthrodesis. $f$ Hand Surg [Am] 1985; 10: 821-9.

15 Nalebuff $E A$. Surgical treatment of tendon rupture in the rheumatoid hand. Surg Clin North Am 1969; 49: 811-22.

16 Nalebuff E A, Patel M R. Flexor digitorum sublimis transfer for multiple extensor tendon rupture in rheumatoid arthritis. Plast Reconstr Surg 1973; 52: 530-3.

17 Ellison M R, Kelly K J, Flatt A E. The results of surgical synovectomy of the digital joints in rheumatoid arthritis. $\mathcal{f}$ Bone foint Surg [Am] 1971; 53: 1041-60.

18 Harrison S H. The importance of the middle (long) finger realignment in ulnar drift. Fournal of Hand Surgery 1976; 1 : 87-91.

19 Straub L R. The rheumatoid hand. Clin Orthop 1959; 15: $127-39$

20 Straub L R. The aetiology of finger deformity in the hand affected by rheumatoid arthritis. Bulletin of the Hospital for Foint Diseases 1960; 21: 322-9.

21 Ellison M R, Flatt A E, Kelly $K$ J. Ulnar drift of the fingers in rheumatoid disease. Treatment by crossed intrinsic transfer. F Bome Foint Surg [Am] 1971; 53: 1061-82.

22 Vahvanen V, Viliakka T. Silicone rubber implant arthroplasty of the metacarpo-phalangeal joint in rheumatoid arthritis: a follow-up study of 32 patients. F Hand Surg [Am] 1986; 11 : 333-9.

23 Bieber W J, Wieland A J, Volenec-Dowling S. Silicone rubber implant arthroplasty of the metacarpophalangeal joints for rheumatoid joints. F Bone foint Surg [Am] 1986 68: 206-9.

24 Swanson A B. Flexible implant arthroplasty for arthritic finger joints. F Bone foint Surg [Am] 1972; 54: 435-55.

25 Wilde A H. Synovectomy of the proximal interphalangeal joint of the finger in rheumatoid arthritis. 7 Bone foint Surg [Am] 1974; 56: 71-8.

26 Ansell B M, Harrison SH, Little H, Thomas B. Synovectomy of the proximal interphalangeal joints. Br $\mathcal{F}$ Plast Surg 1970; 23: 380-5.

27 Ansell B, Harrison S H. A 5 year follow-up of synovectomy of the PIP in rheumatoid arthritis. Hand 1975; 7: 34-6.

28 Soutter $W$ A. The problem of boutonnière deformity. Clin Orthop 1974; 104: 116-33.

29 Nalebuff E A, Millender L H. Surgical treatment of swanneck deformity in rheumatoid arthritis. Orthop Clin North Am 1975; 6: 733-52.

30 Littler J W. Restoration of the oblique retinacular ligamen for correcting hyperextension deformity of the proximal interphalangeal joint. In: Tubiana $\mathrm{R}$, ed. La main rheumacoide. Paris: Expansion Scientifique Francaise, 1969 $155-7$.

31 Granowitz S, Vainio K. Proximal interphalangeal joint arthrodesis in rheumatoid arthritis. Acta Orthop Scand 1966; 37: 301-10.

32 Nalebuff $E$ A Restoration of balance in the rheumatoid thumb. In: Tubiana $R$, ed. La main rheumatoide. Paris: thumb. In: Tubiana R, ed. La main rheumatoide.

33 Harrison S H, Swannell A J, Ansell B M. Repair of extensor pollicis longus using extensor pollicis brevis in rheumatoid arthritis. Ann Rheum Dis 1972; 31: 490-2. 